\title{
Actualización: Marcadores serológicos y clínicos en la predicción del riesgo cardiovascular: ¿nuevos factores de riesgo?
}

Biomarkers and clinical findings in the prediction of cardiovascular risk: novel risk factors?

Sergio Terrasa* $¥$, Gabriela Buela*, Tami Guenzelovich*, Tamara Sigal e Ignacio Vicente Lago* y Fernando Rubinstein ${ }^{\star}$

\begin{abstract}
Resumen
La prevención cardiovascular basada en la valoración del riesgo de cada paciente según su edad y género y sus factores de riesgo tradicionales (tabaquismo, tensión arterial, colesterol total y HDL, glucemia y antecedentes familiares) nos permite categorizar subgrupos y priorizar intervenciones.

Han sido propuestas nuevas formas de ajustar dicha predicción: 1) la clasificación de los pacientes como portadores o no de síndrome metabólico; 2) los niveles de algunos marcadores serológicos como la proteína C reactiva, la hemoglobina glicosilada, la lipoproteína A y la homocisteína 3) la detección de daño asintomático de órgano blanco a través del rastreo de retinopatía, hipertrofia ventricular izquierda y microalbuminuria, y la medición del cociente de la presión arterial entre el tobillo y el brazo, del espesor de la íntima-media carotídea y del puntaje tomográfico de calcio en las arterias coronarias.

Para ser considerados nuevos factores de riesgo estos marcadores deberían permitir reclasificar a una proporción considerable de pacientes desde un riesgo intermedio hacia uno alto o bajo; con la consiguiente modificación de la intensidad de la intervención preventiva y del resultado en salud, además de ser sencillos de medir, económicos, seguros y aceptables.

Sólo la proteina $\mathrm{C}$ reactiva cumple aceptablemente estas últimas condiciones. Sin embargo, consideramos que por ahora la evidencia es insuficiente como para avalar su uso rutinario.
\end{abstract}

\section{Abstract}

Cardiovascular prevention based on the evaluation of individual risk according to age, sex and traditional cardiovascular risk factors (smoking, arterial tension, total and HDL cholesterol, glycemia and family history of cardiovascular disease) allows clinicians to prioritize interventions according to patients risk category.

To improve the risk prediction, different methods have been proposed: 1) classification of patients as having or not metabolic syndrome; 2) determining the serologic levels of $C$ reactive protein, glycated hemoglobin, A lipoprotein and/or homocysteinemia; 3) detection of asymptomatic target organ damage with screening of retinopathy, left ventricular hypertrophy and microalbuminuria, and measurement of arterial pressure ankle-brachial ratio, thickness of carotid intima-media, and calcium tomographic score of coronary arteries.

In addition of being simple to measure, affordable, safe and acceptable; a predictor should allow reclassification of a considerable proportion of patients from an intermediate risk category to a higher or a lower one to be considered a new cardiovascular risk factor, with the consequent modification of the intensity of the preventive intervention and a better outcome.

Based on this premise, only $C$ reactive protein has an acceptable performance; but the authors of the present review conclude that there is still insufficient evidence to support routine use of these potential new cardiovascular risk factor.

Palabras clave: prevención cardiovascular, factores de riesgo cardiovascular. Key words: cardiovascular prevention, cardiovascular risk factors.

Terrasa S, Buela G, Guenzelovich T, Sigal T, Vicente Lago I, Rubinstein F. Bio-marcadores en la predicción del riesgo cardiovascular: ¿nuevos factores de riesgo?. Evid Act Pract Ambul. 14 (1). 12-17.Ene.Mar.2011.

\section{Introducción}

Las enfermedades cardiovasculares (CV) son la principal causa de morbi-mortalidad en todo el mundo. Afectan por igual a ambos sexos y más del $80 \%$ se producen en países en vías de desarrollo. Se calcula que en 2015 morirán cerca de 20 millones de personas por enfermedad CV, sobre todo por cardiopatías y accidentes cerebrovasculares (ACV) previéndose también que seguirá siendo la principal causa de muerte a nivel mundial'. Respecto de Argentina, podemos decir que la tasa de mortalidad por enfermedades CV ajustada por la edad para hombres y mujeres en 2006, fue de 206,4 por 100000 habitantes (265,4 por cada 100000 hombres y 161,8 por cada 100000 mujeres) lo que representa $34,2 \%$ de las muertes y $12,6 \%$ de los años potenciales de vida perdidos (APVP) ese año².

\section{¿Para qué sirve calcular el riesgo cardiovascular de nues- tros pacientes?}

Más del $90 \%$ de los eventos CV se presenta en pacientes que presentan al menos un factor de riesgo, y la presencia de varios factores simultáneamente puede acarrear un mayor riesgo que niveles elevados de uno sólo.

Como ya hemos desarrollado en varios artículos de EVIDEN$\mathrm{ClA}^{3,4,5}$, existe bastante consenso de que es necesario adecuar la intensidad de las intervenciones para la prevención CV al nivel de riesgo de cada individuo, con el objetivo de maximizar su costo-efectividad.
Por ejemplo, las guías de la Organización Mundial de la Salud, así como las guías europeas, norteamericanas y canadienses, y recientemente, la Guía desarrollada por el Ministerio de Salud de Argentina ${ }^{6}$, recomiendan el uso de puntajes para predecir el riesgo de eventos coronarios a diez años con el fin de identificar, modificar y tratar los diferentes factores de riesgo ${ }^{7}$.

Existen numerosas herramientas para estimar el riesgo CV. Entre las más utilizadas, mencionamos la calculadora de "Framingham" (desarrollada en EE.UU.) y la calculadora del "Proyecto SCORE" (desarrollada en Europa) ${ }^{8}$. Sobre los datos de sendas cohortes, ambas derivan de reglas de predicción clínica que permiten clasificar o "discriminar" a los pacientes según su riesgo $\mathrm{CV}$ en tres subgrupos principales: de bajo, intermedio y alto riesgo, que se corresponden aproximadamente con su riesgo de desarrollo de enfermedad CV a diez $a_{n ̃ o s}{ }^{8}$. En líneas generales, estas reglas de predicción clínica incluyen las siguientes variables: el sexo y la edad del paciente, su nivel de colesterol total y HDL, su presión arterial sistólica, la presencia o ausencia de diabetes, y su condición de fumador.

\section{La brecha de detección y el concepto de reclasificación del riesgo cardiovascular}

Se estima que entre 10 y $30 \%$ de los eventos cardiovasculares no pueden ser atribuidos a los factores de riesgo tradicionales, lo que algunos autores denominan "brecha de detección". Este hecho permite suponer la presencia de "nuevos factores de

* Servicio de Medicina Familiar y Comunitaria del Hospital Italiano de Buenos Aires. sergio.terrasa@ hospitalitaliano.org.ar.

‡ Departamento de Salud Pública del Instituto Universitario Hospital Italiano de Buenos Aires. 
riesgo", que explicarían el desarrollo de enfermedad cardiovascular (CV) en ciertos grupos vulnerables. Siguiendo esta línea argumentativa, la detección de estos factores de riesgo, permitirían reclasificar a los pacientes de un nivel de riesgo a otro ${ }^{10}$ con el objetivo de ajustar la intensidad de las intervenciones que pretenden reducir el riesgo $\mathrm{CV}$. Por ejemplo, reclasificar a un pacientes de riesgo intermedio hacia un riesgo alto (para no privarlo de una intervención potencialmente beneficiosa) o desde un riesgo intermedio hacia un riesgo bajo (para evitar someterlo a una intervención innecesaria).

Sin embargo y como aún no queda claro que con estos ajustes se obtengan mejores resultados clínicos, la Fuerza de Tareas Preventivas de EE.UU. (USPSTF) ${ }^{2}$ propone ciertos criterios para considerar un nuevo factor de riesgo como tal, que se describen en el cuadro 1.

Cuadro 1: atributos que debería tener una variable clínica para merecer ser considerada un nuevo factor de riesgo cardiovascular.

1) Debería poder ser medida en forma fácil, segura y no invasiva, y sus resultados tener valores de referencia aceptados.

2) Debería haber una alta prevalencia de valores alterados de esta variable en los individuos con riesgo intermedio.

3) Debería poder ser detectada antes de la aparición de la enfermedad cardiovascular.

4) Debería ser un predictor independiente de enfermedad cardiovascular.

5) Debería lograr reclasificar a una fracción considerable de los individuos de riesgo intermedio.

6) Debería existir un tratamiento disponible para este subgrupo de individuos reclasificados que permita que estos reduzcan su riesgo cardiovascular.

7) Debería contarse con evidencia de que los individuos que fueron asignado a este tratamiento por haber sido reclasificados, tienen mejores resultados que los no tratados.

Modificado por los autores de esta artículo de: Helfand y col. Emerging Risk Factors for Coronary Heart Disease: a summary of systematic reviews conducted for de USPSTF. Ann intern Med 2009;151:496-507.

Agruparemos entonces a estos potenciales nuevos predictores del riesgo $\mathrm{CV}$ en constelaciones clínicas y/o de laboratorio (como el síndrome metabólico), en marcadores serológicos y en marcadores de daño asintomático de órgano blanco.

\section{El sindrome metabólico}

Si bien fue descripto hace unos ochenta años, en las últimas dos décadas se difundió un creciente interés por el síndrome metabólico, para el cual existen varias definiciones ${ }^{11,12,13}$, incluyendo toda una constelación de signos clínicos -relacionados con la obesidad abdominal y con valores de tensión arterial limítrofes o "pre-hipertensivos- y de laboratorio -relacionados con perfiles lipídicos o del metabolismo de la glucosa levemente alterados.

Las características del síndrome metabólico aparecen agrupadas con mayor frecuencia que la esperable por mera asociación casual y tienen una fisiopatogenia con puntos en común (sedentarismo, obesidad abdominal) y alternativas terapéuticas relacionadas con mejoras en el estilo de vida; por lo que consideramos que en algunos pacientes puede ser de utilidad definirlo con el objetivo de promover la adherencia a estos cambios.

Cuando no se considera individualmente la información aporta- da por cada factor de riesgo tradicional en forma separada, los individuos que califican para ser incluidos en este síndrome y comparándolos con quienes no califican, tienen dos a tres veces más riesgo de desarrollar enfermedad $\mathrm{CV}$ y cinco, de desarrollar diabetes ${ }^{14}$. Por ejemplo, un metanálisis publicado en 2007 (que incluyó trabajos con un rango de seguimiento de 2,2 a 18 años) concluyó que si bien el síndrome metabólico se asocia a mayor incidencia de enfermedad CV y muerte (RR de 1,78; IC95\% 1,58 a 2,00) esta asociación es menor cuando se ajusta por los FRCV tradicionales (RR 1,54 con un 95\% IC 1,32 a 1,79$)^{15}$.

Sin embargo, y siendo el síndrome metabólico una constelación de variables con altísima superposición con los llamados factores de riesgo tradicionales, en los últimos años está creciendo el consenso de que, más allá de algunas utilidades clínicas descriptas previamente, esta entidad no aporta mayor capacidad de discriminación en la predicción del riesgo CV global ${ }^{16}$ que las reglas tradicionales de predicción del mismo.

\section{Potenciales nuevos marcadores metabólicos}

\section{Proteína $\mathrm{C}$ reactiva}

Dentro de los marcadores de la inflamación que participan en el proceso de aterogénesis, la proteína $C$ reactiva $(P C R)$ es el más estudiado ${ }^{17,18,19}$

Ya habíamos comentado en "EVIDENCIA" varios estudios que han documentado su capacidad predictiva, por ejemplo sobre el riesgo de desarrollar accidente cerebrovascular isquémico ${ }^{20}$ Tiene buena correlación con los niveles plasmáticos de colesterol $\mathrm{LDL}^{21}$ (ver tabla 1) y sería un predictor de eventos cardiovasculares (RR de mortalidad CV a ocho años: 1,55; IC95\% 1,37 a 1,76) representando un subgrupo de alto riesgo los pacientes con LDL menor $130 \mathrm{mg} / \mathrm{dL}$ y $\mathrm{PCR}$ mayor a $3 \mathrm{mg} / \mathrm{L}$.

Tabla 1: correlación entre los niveles plasmáticos de proteína $C$ reactiva y colesterol LDL.

\begin{tabular}{c|c}
\hline Proteina C reactiva & Colesterol LDL \\
\hline Menor $1 \mathrm{mg} / \mathrm{L}$ & Menor $130 \mathrm{mg} / \mathrm{dL}$ \\
\hline 1 a $3 \mathrm{mg} / \mathrm{L}$ & $130-160 \mathrm{mg} / \mathrm{dL}$ \\
\hline Mayor $3 \mathrm{mg} / \mathrm{L}$ & Mayor $160 \mathrm{mg} / \mathrm{dL}$ \\
\hline
\end{tabular}

Fuente: Ridker P y col. Comparison of C-Reactive Protein and LowDensity Lipoprotein Cholesterol Levels in the Prediction of First Cardiovascular Events. N Engl J Med 2002; 347:1557-1565.

El estudio Júpiter ${ }^{22}$ fue un ensayo clínico aleatorizado (ya comentado en la revista "EVIDENCIA") que había incluido pacientes de 60 a 70 años con niveles de colesterol LDL menores a $130 \mathrm{mg} / \mathrm{dL}$ y de PCR mayores a $2 \mathrm{mg} / \mathrm{L}$. Los pacientes de la rama intervención habían recibido $20 \mathrm{mg}$ de rosuvastatina durante una mediana de 18 meses y un máximo de cinco años. Esta intervención se asoció a una disminución de $50 \%$ del colesterol LDL y del $37 \%$ de los niveles de PCR y a una reducción estadísticamente significativa de la mortalidad (HR 0,8; IC 95\% 0,67 a 0,97) y de la ocurrencia de un evento CV combinado** (HR 0,56; 0,46 a 0,69).

Sobre la base de estos y otros resultados, se planteó la existencia de una respuesta diferente a las estatinas de acuerdo al "estado inflamatorio" del paciente, evaluado por el nivel de PCR, sugiriendo que serían aún más beneficiosas en aquellos pacientes que presentaran niveles elevados de PCR. 
Un subanálisis del Heart Protection Study ${ }^{24}$ (HPS) recientemente publicado, evaluó específicamente esta hipótesis. Los autores aleato-rizaron a más de 20.000 hombres y mujeres de 40 a 80 años de edad con alto riesgo vascular a recibir $40 \mathrm{mg}$ de simvastatina o placebo, y planificaron un análisis de seis subgrupos de acuerdo a los niveles de PCR (desde menos de 1,25 hasta más de $8 \mathrm{mg} / \mathrm{L}$ ). Los resultados mostraron una reducción del 25 al $28 \%$ en el riesgo de eventos CV mayores en el grupo tratado (comparado con placebo), y este efecto fue similar en todos los sub-grupos de PCR. Si bien los participantes del HPS tenían un riesgo vascular mayor que los del Jupiter, este estudio reportó que la reducción de riesgo fue observada incluso en aquellos pacientes con niveles de PCR menores a $1,25 \mathrm{mg} / \mathrm{L}$ y de LDL menores a $130 \mathrm{mg} \%$. Estos hallazgos, explicados mayormente por la reducción del nivel de LDL, no parecen sustentar la hipótesis de efectos diferentes del tratamiento con estatinas en relación con la PCR como marcador de estado inflamatorio.

Más allá de la controversia descripta previamente, la determinación de los niveles de PCR permitiría reclasificar a un subgrupo (cercano al 50\%) de las mujeres con riesgo intermedio a través del puntaje de Reynolds ${ }^{25}$ que fue diseñado para predecir el riesgo de presentar un evento CV a diez años e incluye entre sus variables a la edad, a la tensión sistólica, a los niveles de colesterol total y HDL, a la condición de fumador, a los niveles de PCR y a los antecedentes de eventos CV en familiares de primer grado menores de 60 años.

Por lo tanto y si bien todavía falta información para poder sacar conclusiones contundentes, la PCR sería la variable con mejor cumplimiento de los criterios para ser considerada un nuevo factor de riesgo CV ya que existe moderada evidencia de que permitiría reclasificar a algún subgrupo de pacientes de bajo riesgo CV basal y que esta reclasificación permitiría incluirlos en algún tratamiento beneficioso para disminuir su riesgo CV.

\section{Hemoglobina glicosilada}

Además de su conocida asociación con el riesgo de desarrollo de enfermedad micro y macrovascular en personas con diabetes; y como comentáramos en el número pasado de EVI$D_{E N C I}{ }^{26}$ en individuos sin diabetes ${ }^{\dagger \dagger}$, los niveles de hemoglobina glicosilada ( $\mathrm{HbA1c}$ ) se asocian con el riesgo a 11 años de enfermedad coronaria, de accidente cerebrovascular y de muerte, independientemente de los niveles de glucemia en ayunas y de los factores de riesgo tradicionales ${ }^{27}$. Ver tabla 2.

Tabla 2: asociación entre los niveles de hemoglobina glicosilada y el riesgo cardiovascular luego de ajustar por los factores de riesgo tradicionales.

\begin{tabular}{|c|c|c|c|c|c|}
\hline & $\begin{array}{c}\text { Menor } \\
\text { a 5\% }\end{array}$ & $\begin{array}{l}5,0 a \\
5,5 \%\end{array}$ & $\begin{array}{c}5,5 a \\
6 \%\end{array}$ & $\begin{array}{c}6 \mathbf{a} \\
6,5 \%\end{array}$ & $\begin{array}{c}\text { Mayor a } \\
6,5 \%^{\mathrm{a}}\end{array}$ \\
\hline & 0,95 & 1,00 & 1,25 & 1,88 & 2,46 \\
\hline a 11 años & $(0,73$ a 1,22$)$ & & $(1,09$ a 1,44$)$ & $(1,55$ a 2,28$)$ & $(1,84$ a 3,28$)$ \\
\hline
\end{tabular}

aDiagnóstico de diabetes según American Diabetes Association. Diagnosis and classification of diabetes mellitus. Diabetes Care 2010;33:Suppl 1:S62-S69. Fuente: Selvin E et al. Glycated Hemoglobin, Diabetes, and Cardiovascular Risk in Nondiabetic Adults. N Engl J Med 2010;362:800-11.

Comparada con la glucemia en ayunas, la $\mathrm{HbA} 1 \mathrm{c}$ presenta como principales ventajas, una mayor reproducibilidad y menor variabilidad interindividual y no requerir que el paciente se encuentre en ayunas. Sin embargo, tiene limitado valor en pacientes con anemia, todavía existe variabilidad en sus resultados y no todos los centros cuentan con la posibilidad de determinarla ${ }^{28}$.

Todavía queda sin respuesta si llegará el momento de agregar este parámetro a la reglas de predicción de riesgo cardiovascular con el objetivo de refinar la estimación del mismo.

\section{Lipoproteína A}

El más grande de los estudios retrospectivos ${ }^{29}$ hasta el momento, un estudio casos y controles anidado en el estudio INTERHEART $^{30}$ (ambos ya comentados en "EVIDENCIA" que la Lipoproteína $A(L p A)$ tendría mayor poder predictivo que el cociente colesterol total/colesterol HDL.

La LpA tiene una estructura similar al colesterol LDL ${ }^{33,34}$, considerándose a los valores plasmáticos mayores de $30 \mathrm{mg} / \mathrm{dL}$ un factor de riesgo $\mathrm{CV}$ independiente para el desarrollo de enfermedad coronaria a los seis años (OR 1,1; IC95\% 1,07 a 1,16) ${ }^{35}$.

Algunos expertos recomiendan intensificar el tratamiento (p. ej. incluir ácido nicotínico) si la concentración de la LpA está aumentada en pacientes con enfermedad CV establecida, con historia familiar de enfermedad CV en ausencia de dislipemia o ante una persona con hipercolesterolemia refractaria a fármacos que disminuyen el LDL.

Sin embargo, no hay evidencia de que disminuir sus niveles reduzca los eventos $\mathrm{CV}$, ni si permite reclasificar a los pacientes según su riesgo, por lo que no está indicado realizar rastreo rutinario ${ }^{2}$

\section{Homocisteinemia}

La homocisteína es un aminoácido intermediario derivado de la conversión de metionina a cisteína. Por otro lado, la homocistinuria es una enfermedad autosómica recesiva caracterizada por elevaciones plasmáticas y urinarias de la homocisteína, habiéndose evidenciado un aumento de los fenómenos tromboembólicos y aterogénicos en quienes padecen este trastorno ${ }^{36}$

La mayor parte de los datos sugieren que la hiperhomocisteinemia moderada es un factor de riesgo independiente para la enfermedad CV y el tromboembolismo recurrente ${ }^{37,38}$. Por otro lado, existe evidencia que la suplementación de ácido fólico y vitamina B12 se asocian a una disminución de la homocisteine$\mathrm{mia}^{39}$.

Esta argumentación condujo a ensayar su administración con el objetivo de prevenir eventos CV, lo que no se ha comprobado en los estudios. Por ejemplo, un meta-análisis de ocho ensayos clínicos (n: 37485) que habían comparado suplementos de acido fólico contra placebo en pacientes con riesgo $\mathrm{CV}$ elevado, evidenció que si bien el suplemento con ácido fólico se asoció a una reducción de un $25 \%$ en los niveles plasmáticos de homocisteína, no hubo diferencias significativas entre los grupos en la tasa de eventos CV mayores (RR: 1,01; IC95\% $0,97$ a 1,05$)$ eventos coronarios $(1,03 ; 0,97$ a 1,10$)$ y accidente cerebrovascular $0,96(0,87$ a 1,06$)$ luego de cinco años de seguimiento ${ }^{40}$.

En consecuencia podemos concluir que existe fuerte evidencia en contra de realizar rastreo de hiperomocisteinemia y/o de administrar suplementos vitamínicos con el objetivo de prevenir la enfermedad CV (aún en pacientes de alto riesgo).

"O por lo menos que no cumplen todavía los criterios como para ser calificados como diabéticos. 


\section{Marcadores de enfermedad cardiovascular subclínica o de} daño asintomático de órgano blanco

\section{Hipertrofia del ventrículo izquierdo}

La hipertrofia del ventrículo izquierdo (HVI) es un hallazgo frecuente en pacientes con hipertensión y puede ser diagnosticado por electrocardiograma (ECG) o ecocardiografía ${ }^{41}$, siendo éste último un método más sensible para su detección.

La presencia de HVI se asocia con un aumento en la incidencia de insuficiencia cardíaca, arritmias ventriculares, muerte por infarto de miocardio, disminución de la fracción de eyección del VI, muerte súbita, dilatación de la raíz aórtica y eventos cerebrovasculares ${ }^{42}$.

El aumento en el riesgo CV está directamente relacionado con el grado de aumento de la masa ventricular izquierda ${ }^{43,44}$ siendo este efecto independiente de la tensión arterial ${ }^{45}$.

En una cohorte de 1033 individuos mayores de 50 años con hipertensión arterial esencial y sin eventos cardiovasculares previos que habían sido seguidos durante una media de tres años se evidenció que la tasa de eventos CV fue significativamente mayor en el $29 \%$ de los pacientes que tenían mayor masa ventricular (más de $125 \mathrm{~g} / \mathrm{m}^{2}$ de superficie corporal). Luego del ajuste por los otros factores de riesgo CV, la HVI se asoció con un aumento de eventos cardiovasculares (RR: 2,08 IC95\% 1,22 a 3,57) por cada aumento de $39 \mathrm{~g} / \mathrm{m}^{2}$ de la masa ventricular ${ }^{14}$

Sin embargo el riesgo CV asociado a la HVI puede ser reducido por el tratamiento antihipertensivo ${ }^{\ddagger}$, que genera una regresión de la misma ${ }^{46}$ y se asocia a una reducción de aproximadamente $10 \%$ de la masa ventricular. La regresión de la HVI continúa a lo largo del tiempo (tres años o más) pudiendo llegar a ser completa ${ }^{47}$. A su vez, la evidencia surgida de estudios observacionales sugiere que la regresión de la HVI (electrocardiográfica o ecográfica) se asocia con una reducción en el riesgo cardiovascular, estando el beneficio directamente relacionado con el grado de reducción la masa ventricular ${ }^{48,49}$.

Vale destacar que el rastreo de HVI forma parte de la evaluación rutinaria propuesta por la mayoría de las guías de práctica clínica ${ }^{50,51,52,53}$ para el abordaje del paciente con hipertensión arterial.

\section{Retinopatía}

La retinopatía está fuertemente asociada a presencia de hipertensión arterial y a la de diabetes y es objetivable mediante la realización de un examen de fondo de ojo por un operador entrenado.

Las anormalidades de la microcirculación de la retina son consideradas un factor de riesgo $\mathrm{CV}$ independiente. Aunque los mecanismos exactos de ésta asociación no son claros, hay indicios de que ciertas anomalías de la retina (como microaneurismas y hemorragias) pueden predecir el riesgo de accidentes cerebrovasculares y de enfermedad coronaria ${ }^{54,55}$. Por ejemplo, un estudio de casos y controles basado en la población del estudio Beaver Dam Eye ${ }^{56}$ reportó que las alteraciones en el diámetro en la zona de bifurcación arteriolar $(p<0,02)$ y la disminución de tortuosidad de las arteriolas retinianas se asocian al aumento del riesgo de muerte por cardiopatía isquémica $(p<0,011)$; mientras que el aumento de la longitud arteriolar y el estrechamiento arteriolar generalizado implican una mayor mortalidad por accidente cerebrovascular $(p<0.02)$ perdiendo poder predictivo luego de ajustar por los valores de tensión arterial sistólica $(p<0,15)^{57}$.

Recordamos que el rastreo rutinario de retinopatía forma parte de la evaluación habitual de los pacientes con hipertensión arterial y con diabetes propuesta por los principales consensos de expertos ${ }^{48,49,58}$.

\section{Microalbuminuria}

La cuantificación de la microalbuminuria se utiliza rutinariamente para el rastreo de daño renal subclínico en los pacientes con diabetes ${ }^{56}$. Como hemos comentado en EVIDENCIA ${ }^{59}$, diferentes estudios poblacionales en personas sin diabetes y sin hipertensión documentaron su asociación con la progresión de nefropatía y con el riesgo de desarrollar enfermedad CV, comportándose como un factor de riesgo CV independiente ${ }^{60,61}$. Ver tabla 3.

Tabla 3: tasa ajustada ${ }^{a}$ de mortalidad, por cada 1000 personas año, según el nivel de filtrado glomerular y proteinuria medida por tiras reactivas.

\begin{tabular}{c|c|c|c} 
& \multicolumn{3}{|c}{$\begin{array}{c}\text { Nivel de proteinuria y riesgo relativo de mortalidad } \\
\text { (promedio de } \mathbf{3} \text { años de seguimiento y riesgo absoluto } \mathbf{3} \% \text { ) }\end{array}$} \\
\hline $\begin{array}{c}\text { Filtrado } \\
\text { glomerular }\end{array}$ & Normal & Leve & Severa \\
\hline$>\mathbf{6 0}$ & 2,7 & 5,8 & 7,2 \\
& $(2,6 \mathrm{a} 2,8)$ & $(5,5 \mathrm{a} 6,0)$ & $(6,6 \mathrm{a} 7,8)$ \\
\hline $\mathbf{4 5}$ a $\mathbf{5 9}$ & 2,9 & 5,2 & 7,2 \\
& $(2,7 \mathrm{a} 3,0)$ & $(4,9 \mathrm{a} 5,5)$ & $(6,5 \mathrm{a} 7,8)$ \\
\hline $\mathbf{3 0}$ a $\mathbf{4 4}$ & 4,0 & 5,8 & 7,5 \\
& $(3,7$ a 4,2) & $(5,4 \mathrm{a} 6,2)$ & $(6,8 \mathrm{a} 8,2)$ \\
\hline $\mathbf{1 5}$ a $\mathbf{2 9}$ & 6,7 & 9,1 & 10,4 \\
& $(6,2 \mathrm{a} 7,3)$ & $(8,2 \mathrm{a} 10,0)$ & $(9,3 \mathrm{a} 11,6)$ \\
\hline
\end{tabular}

ajustado por edad, sexo, hipertensión arterial, nivel socioeconómico, antecedentes de cáncer, enfermedad cerebrovascular, insuficiencia cardiaca, enfermedad pulmonar obstructiva crónica, demencia, diabetes, HIV/SIDA, tumor metastático sólido, infarto de miocardio, hepatopatia, enfermedad ulcerosa péptica, enfermedad vascular periférica, enfermedad renal y enfermedad reumática.

Resumido de: Hemmelgayny col. Relation between kidney, proteinuria and adverse outcomes. JAMA 2010; 303 (5): 423-9.

El valor de la micro albuminuria y el nivel de deterioro del filtrado glomerular se asocian directamente con la incidencia de eventos CV y con el riesgo de muerte, y las Guías de práctica y consensos de expertos recomiendan su rastreo en hiperten$\operatorname{sos}^{49,50}$.

Si bien existe evidencia que justifica la utilización de antihipertensivos de la familia de Ios IECA o los ARA II para disminuir la progresión de nefropatía y disminuir el riesgo CV en los pacientes diabéticos con micro-albuminuria ${ }^{62}$; no existe aún información que respalde este tipo de tratamiento en individuos de la población general (sin diabetes ni hipertensión) para reducir la incidencia de eventos CV o la mortalidad, por lo que tampoco está recomendado rastrear esta condición clínica.

\section{Espesor de la íntima-media carotídea}

Se sabe que el engrosamiento del espesor de la íntima-media carotídea (EIMC) medido a través de una ecografía doppler, se

\# Este fenómeno se da con el uso de inhibidores de la enzima convertidora de la angiotensina (IECA) con antagonistas de los receptores de la angiotensina II (ARA II) con inhibidores de la renina, con bloqueantes cálcicos (diltiazem, verapamilo y amlodipina) con alfa-metildopa y con alfa-bloqueantes. 
asocia a los mecanismos que causan aterosclerosis ${ }^{63}$.

Si bien este estudio diagnóstico tiene las ventajas de ser repetible, no invasivo y seguro (no utiliza radiación) tiene la desventaja de que sus mediciones no se encuentran tan estandarizadas y muchas veces los resultados pueden variar entre distintos observadores. Por otro lado, sólo permite observar las arterias carótidas y no las coronarias, que son responsables de la mayoría de las muertes por enfermedad cardiovascular, no quedando claramente establecido cuán fuerte es la asociación entre la detección de aterosclerosis diagnosticada en las carótidas y la presente en las arterias coronarias ${ }^{3}$.

En una cohorte prospectiva de 4000 participantes de 45 a 65 años, se observó que cada aumento de un milímetro de EIMC se asociaba con un aumento de dos a cinco veces del riesgo de infarto agudo de miocardio (IAM) y de cuatro a ocho veces del de accidente cerebrovascular (ACV) incluso luego de ajustar por los factores de riesgo CV tradicionales ${ }^{64}$; mientras que en un metanálisis ${ }^{65}$ de ocho estudios observacionales se documentó que el aumento en $0,1 \mathrm{~mm}$ de EIMC se asocia a un HR de 1,15 (IC95\% 1,12 a 1,17) para IAM y de 1,18 (IC95\% 1,16 a 1,21) para ACV

Algunos consensos ${ }^{3}$ recomiendan considerar la medición del EIMC en pacientes con antecedentes familiares de ECV temprana, en menores de 60 años con alteración severa de un solo FRCV no candidatos a fármacos o en mujeres menores de 60 con dos FRCV.

\section{Puntaje (score) tomográfico de calcio}

Se obtiene de determinación tomográfica que no requiere el uso de medios de contraste. Se evalúa el puntaje de Agatston, que va de 0 a 400 y que sería un predictor independiente de eventos coronarios ${ }^{66}$. Sin embargo, es un método diagnóstico que implica recibir radiación; y que además de ser costoso y poco accesible, fue evaluado en trabajos de débil calidad metodológica.

Si bien algunos consensos de expertos recomiendan medirlo en pacientes con antecedentes familiares de enfermedad $\mathrm{CV}$ precoz o en los de riesgo intermedio ( $\mathrm{ACC}-\mathrm{AHA}$ ) no existe evidencia que avale esta indicación, especialmente en población de bajo y moderado riesgo ${ }^{2}$.

\section{Indice tobillo brazo}

Se considera un "equivalente coronario"67 a padecer enfermedad vascular periférica (EVP) sintomática, ya que su presencia se asocia a un riesgo de muerte $\mathrm{CV}$ de $3 \%$ al año ${ }^{68}$. El índice tobillo brazo de presión arterial (ITBPA) se obtiene dividiendo la presión arterial sistólica obtenida en el tobillo (arteria tibial posterior o pedia) con la obtenida en el brazo ${ }^{16}$. Un valor menor a 0,9 tiene alta especificidad (98\%) y sensibilidad $(82 \%)$ para el diagnóstico de enfermedad arterial periférica, valores entre 0,9 y 1,1 se consideran en el límite, y valores mayores a 1,1 normales. Constituye un procedimiento de bajo riesgo y accesible. Si bien clásicamente ha sido realizado y validado a través de la medición por ecografía doppler, también tendría buen rédito diagnóstico cuando se lo valora por palpación, como hemos discutido en otro número de "EVIDENCIA"69.

Un discutido metanálisis comentado en "EVIDENCIA"16 y que incluyó el seguimiento de 16 cohortes poblacionales que habían incluido 480.325 personas/año, sugiere que mejoraría la predicción del riesgo en cerca del $10 \%$ de las mujeres de mediana edad de bajo (menor a $10 \%$ de riesgo de eventos CV a los diez años) y moderado (10 a $20 \%$ ) riesgo, en términos de ubicarlas en una categoría de mayor riesgo (mayor a $20 \%$ ). Ver tabla 4.
Tabla 4: reclasificación de un subgrupo (24\%) de mujeres que habían sido categorizadas como de bajo y moderado riesgo de acuerdo al puntaje de Framingham hacia "alto riesgo" luego de ajustar la predicción con los resultados de índice tobillo-brazo de presión arterial.

\begin{tabular}{c|c|c}
\hline \multirow{2}{*}{} & \multicolumn{2}{|c}{$\begin{array}{c}\text { Riesgo cardiovascular a diez añosa en mujeres } \\
\text { de mediana edad }\end{array}$} \\
\cline { 2 - 3 } & $\begin{array}{c}\text { Luego de categorizarlas } \\
\text { con el puntaje de } \\
\text { Framingham }\end{array}$ & $\begin{array}{c}\text { Luego de ajustar la } \\
\text { predicción anterior por un } \\
\text { ITBPA (menor a 0,9) }\end{array}$ \\
\hline Alto & $27 \%(1418)$ & $44 \%(200)$ \\
\hline Moderado & $13 \%(5563)$ & $25 \%(558)$ \\
\hline Bajo & $11 \%(15505)$ & $21 \%(1083)$ \\
\hline
\end{tabular}

ITBPA: índice tobillo brazo de presión arterial. aMortalidad total, mortalidad CV y eventos coronarios mayores.

Podemos ver que $558(9,97 \%)$ de las 5563 pacientes que habían sido clasificadas inicialmente como de moderado y 1083 (14,32\%) de las 15505 que habían sido clasificadas en el subgrupo de bajo riesgo fueron re-categorizadas hacia al subgrupo de alto riesgo. Al producirse este cambio de categorización también cambiará la intensidad de la intervención de reducción de riesgo cardiovascular a la que van a ser sometidas. Fuente: Ankle Brachial Index Collaboration. Ankle Brachial Index Combined With Framingham Risk Score to Predict Cardiovascular Events and Mortality. JAMA 2008; 300: 197-208.

Sin embargo, luego de observar algunos problemas metodológicos del citado metanálisis, la Fuerza de Tareas Preventivas de EE.UU. ${ }^{2}$ concluye que no existe evidencia suficiente como para solicitarlo en forma rutinaria.

\section{Conclusiones}

Como dijimos al principio de este artículo, toda variable clínica que aspire a ser considerada un nuevo factor de riesgo $\mathrm{CV}$ debería: 1) poder ser detectada antes de la aparición de enfermedad CV clínicamente manifiesta; 2) medirse en forma sencilla, económica, segura y no invasiva, y poseer valores de referencia aceptados; 3) existir una moderada /alta prevalencia de valores alterados en la subpoblación de riesgo CV intermedio y ser un predictor independiente de enfermedad CV, que logre reclasificar el riesgo de una fracción considerable de esta subpoblación; y 4) lograr que los individuos reclasificados reduzcan su riesgo $\mathrm{CV}$ luego de que reciban un tratamiento disponible que no se les habría prescripto si no hubieran sido reclasificados. Esto implica que debería contarse con evidencia de que los individuos asignados a este tratamiento por haber sido reclasificados, tienen mejores resultados que los no tratados.

Dada la evidencia actualmente disponible, consideramos que el único potencial candidato para constituirse en el futuro en un marcador que nos ayude a refinar la predicción del riesgo $\mathrm{CV}$ de la sub-población de riesgo intermedio es el dosaje de PCR. Vale destacar que un solo trabajo ${ }^{23}$ ha demostrado que el dosaje de PCR permite seleccionar a un subgrupo de pacientes que no habría sido identificado través de la categorización de riesgo basada solamente en los factores de riesgo CV tradicionales, y que este subgrupo de beneficia de un tratamiento intensivo con estatinas; por lo que su pesquisa podría comenzar a considerarse en algunos pacientes de riesgo moderado (ej. antecedentes familiares de ECV temprana) para ajustar la intensidad del tratamiento farmacológico.

Como conclusión general, podemos resumir que existe escasísima evidencia de que estos potenciales nuevos factores de riesgo sobre los que hemos discutido tengan un rol rele- 
vante en la toma de decisiones en los pacientes en prevención primaria, ya que la mayoría de estos marcadores no logran recategorizar a los pacientes de riesgo intermedio, y de los que lo logran, un solo ensayo clínico ha demostrado que esta recategorización del riesgo redunda en beneficios clínicamente significativos. No hay que olvidar los posibles daños que estas recategorizaciones podrían producir (fenómenos de etiquetamiento, aumento de la incidencia de efectos adversos), los costos derivados de este tipo de estrategia que suelen conducir a tratamientos más intensivos; y que como podemos ver en la tabla 5, la subcategoría de riesgo intermedio solo representa entre el 2,9 (Barquisimeto, Venezuela) y el 6\% (Santiago de Chile) de la población de nuestra región ${ }^{71}$.
Tabla 5: porcentaje de la población con riesgo cardiovascular intermedio en siete regiones de América, de acuerdo al puntaje de Framingham y definido como riesgo de infarto agudo de miocardio, angina de pecho o muerte coronaria.

\begin{tabular}{c|c|c|c|c|c|c}
\hline $\begin{array}{c}\text { Barquisimeto } \\
\text { (Venezuela) }\end{array}$ & $\begin{array}{c}\text { Bogotá } \\
\text { (Colombia) }\end{array}$ & $\begin{array}{c}\text { Buenos Aires } \\
\text { (Argentina) }\end{array}$ & $\begin{array}{c}\text { Lima } \\
\text { (Perú) }\end{array}$ & $\begin{array}{c}\text { México } \\
\text { (DF) }\end{array}$ & $\begin{array}{c}\text { Quito } \\
\text { (Ecuador) }\end{array}$ & $\begin{array}{c}\text { Santiago } \\
\text { (Chile) }\end{array}$ \\
\hline $2,9 \%$ & $4,5 \%$ & $5 \%$ & $4,1 \%$ & $3,8 \%$ & $5,5 \%$ & $6 \%$ \\
\hline
\end{tabular}

Fuente: Schargrodsky H. CARMELA: Assessment of Cardiovascular Risk in Seven Latin American Cities. American Journal of Medicine (2008) 121, 58-65.

Recibido el 30/10710 y aceptado el 28/12/10.

Referencias

1.Brindle P y col. Predictive accuracy of the Framingham coronary risk score in British men: prospective cohort study. BMJ 2003; 327:1267 (29 November) doi:10.1136/bmj.327.7426.1267

2. Rubinstein A y col. Estimación de la carga de las enfermedades cardiovasculares atribuible a factores de riesgo modificables en Argentina. Rev Pana
3. Kopitowski K y col. Riesgo vascular global (primera parte).Evidencia en Atención Primaria. Vol 6 No5.Sept-Octubre de 2003.Pag 152. Nov.de 2003.

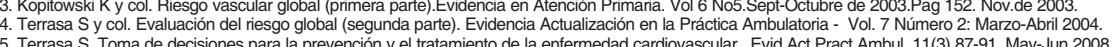

5. Ministerio de Salud de la Nación (Argentina). Presidencia de la Nación. Prevención de las Enfermedades Cardiovasculares. Guía de Bolsillo para la estimación y el manejo del riesgo cardiovascular. 2009 . Ministerio de Salud

de la Nación

7. van Steenkiste B y col. Systematic review of implementation strategies for risk tables in the prevention of cardiovascular diseases, 2008 June; 4(3): 535-545

7. van Steenkiste B y col. Systematic review of implementation strategies for risk tables in the prevention of cardiovascular diseases, 2008 June; 4(3): 535-545.
8. Cooney M y col. Cardiovascular Risk-Estimation Systems in Primary Prevention Do They Differ? Do They Make a Difference? Can We See the Future? Circulation. 2010;122:300-310.

9. Gerber T. Carotid Intima- Media thickness: can it close the dettection gap for cardiovascular risk? Mayo Clinic Proc. 2009; 84(3)218-220.

10. Helfand y col. Emerging Risk Factors for Coronary Heart Disease: a summary of systematic revies confucted for de USPSTF. Ann intern Med 2009; 151:496-507.

11. Alberti K y col. Definiton, diagnosis and classification of diabetes mellitus and its complications. I: diagnosis and classification of diabetes mellitus. Provisional report of a WHO consultation. Diabet Med 1998;15:539-53.

12. Grundy S y col. Diagnosis and management of the metabolic syndrome. An American Heart Association/National Heart, Lung and Blood Institt
13. Alberti K y col. Epidemiology Task Force Consensus Group. The metabolic syndrome, a new world wide definition. Lancet 2005;366:1059-62.
14. Wilson P y col. Metabolic syndrome as a precursor of cardiovascular disease and type 2 diabetes mellitus. Circulation 2005; 112:3066-72.

15. Gami A y col. Metabolic syndrome and risk of incident cardiovascular events and death: a systematic review and meta-analysis of longitudinal studies. J Am Coll Cardiol. 2007 Jan 30;49(4):403-14.

16. Bayturan O y col.The Metabolic syndrome, its component risk factors, and progression of coronary atherosclerosis. Arch Intern Med. 2010 Mar 8;170(5):478-84.

16. Bayturan O y col.The Metabolic syndrome, its component risk factors, and progression of coronary atherosclerosis. Arch Intern Med. 2010 Mar 8;170(5):478-84.
17. Danesh J, et al. Low grade inflammation and coronary heart disease: prospective study and updated meta-analyses. BMJ 2000;321:199-204, doi:10.1136/bmj.321.7255.199.

18. Ridker P y col. Clinical Application of C- Reactive Protein for Cardiovascular Disease Detection and Prevention. Circulation 2003;107:363-369.

19. The Emerging Risk Factors Collaboration. C-reactive protein concentration and risk of coronary heart disease, stroke, and mortality: an individual participant meta-analysis. The Lancet: 375 (9709) 132-140, 9 January 2010. 20. Bauso D. La Proteína C Reactiva y la ateromatosis carotídea son factores de riesgo independientes de ACV isquémico. Evid Act Pract Ambul. Comentado de: Cao J y col. C-Reactive Protein, Carotid Intima-Media Thicknes and Incidence of Ischemic Stroke in the Elderly. The Cardiovascular Health Study. Circulation. 2003; 108: 166-170. Disponible en URL: hittp://www.foroaps.org/files/proteina\%20c\%20y\%20acv.pdf (último acceso 21/11/10). 21. Ridker P y col. Comparison of C-Reactive Protein and Low-Density Lipoprotein Cholesterol Levels in the Prediction of First Cardiovascular Event
22. JUPITER Study Group Rosuvastatin to Prevent Vascular Events in Men and Women with Elevated C-Reactive Protein. NEJM 2008; 359:21.

23. Tesolín P. La rosuvastatina previene eventos cardiovasculares en personas con elevación de la proteina C reactiva. Evid Act Pract Ambul. 12(1): 11. Ene-Mar, 2009. Comentado de Ridker P y col. Rosuvastatin to prevent vascular events in men and women with elevated C-reactive protein N Engl J Med. 2008 Nov 20; 359(21):2195-207. Disponible en URL: http://www.foroaps.org/files/rosu_proteina_c.pdf (último acceso 21/11/10).

vascular events in men and women with elevated C-reactive protein N Engl J Med. 2008 Nov 20; $359(21): 2195-207$. Disponible en URL: http://www.foroaps.org/files/rosu_proteina_c.pdf (uiltimo acceso 21/11/10).
24. Heart Protection Study Collaborative Group. C-reactive protein concentration and the vascular benefits of statin therapy: an analysis of 20536 patients in the Heart Protection Study. The Lancet, Early Online Publication, 28

January 2011 doi:10.1016/S0140.6736(10)62174.5
25. Ridker P y col. Development and Validation of Improved Algorithms for the Assessment of Global Cardiovascular Risk in Women. JAMA. 2007;297:611-619.

26. Sigal T. La hemoglobina glicosilada tambien predice el riesgo cardiovascular en pacientes sin diabetes. Evid Act Pract Ambul. 13(3). 93. Jul-Sept 2010. Comentado de: Selvin E, Steffes M, Zhu H et al. Glycated Hemoglobin, Diabetes, and Cardiovascular Risk in Nondiabetic Adults. N Engl J Med 2010;362:800-11

27. Selvin E et al. Glycated Hemoglobin, Diabetes, and Cardiovascular Risk in Nondiabetic Adults. N Engl J Med 2010;362:800-11.

28. Sociedad Argentina de diabetes. Estandarizacion de HibA1c. Mainetti H. 2009. Vol a3. Nro3.
29. McQueen M y col. ipids, lipoproteins, and apolipoproteins as risk markers of myocardial infarction in 52 countries (the INTERHEART study): a case-control study. Lancet 2008:372(9634):224-33.
30. Yusuf S y col. Effect of potentially modifiable risk factors associated with myocardial infarction in 52 countries (the INTERHEART study): case-control study. Lancet 2004; 364:937-52.

31. Ciapponi A. Las apolipoproteínas predicen mejor riesgo el cardiovascular que el colesterol. Evid. actual. práct. ambul; 11(5): 141, Sep-Oct.2008. McQueen MJ y col. Lipids, lipoproteins, and apolipoproteins as risk markers of myocardial infarction in 52 countries (the INTERHEART study): a case-control study. Lancet 2008;372(9634):224-33. Disponible en URL: http://www.foroaps.org/files/141.pdf (último acceso 21/11/10).

32. Navarro M, Capellato N, Wurcel V. Los factores de riesgo modificables en la enfermedad cardiovascular son similares en 52 países. Evid. Act. Práct. Ambul. 2005;14-15. Comentado de: Yusuf S y col. Effect of potentially modifiable risk factors associated with myocardial infarction in 52 countries (the INTERHEART study): case-control study. Lancet 2004; 364:937-52.

El estrés psicosocial incrementa el riesgo de IAM. Comentado de: Rosengren A y col. Association of psychosocial risk factors with risk of actue myocardial infraction in 11119 cases and 13648 controls from 52 countries (the INTERHEART study):case-control study. Lancet 2004; 364:953 -962. Disponible en URL: http://www.foroaps.org/files/2005_8_1_14\%2015.pdf (último acceso 21/11/10)

33. Bennet A y col. Lipoprotein(a) levels and risk of future coronary heart disease: large-scale prospective data. Arch Intern Med. 2008; 2008168(6):598.

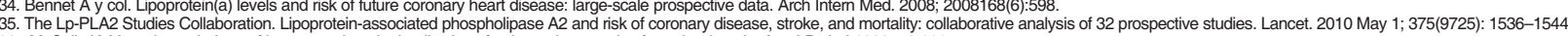

36. McCully K. Vascular pathology of homocysteinemia: Implications for the pathogenesis of arteriosclerosis. Am J Pathol 1969; 56:111.

37. Ueland P y col. Plasma homocysteine, a risk factor for vascular disease: Plasma levels in health, disease, and drug therapy. J Lab Clin Med 1989; 114:473.

38. McCully K. Homocysteine and vascular disease. Nat Med 1996; 2:386.

39. Appel $L$ y col. Effect of dietary patterns on serum homocysteine: results of a randomized, controlled feeding study. Circulation 2000; $102: 852$.

40. Clarke R y col. Effects of Lowering Homocysteine Levels With B Vitamins on Cardiovascular Disease, Cancer, and Cause-Specific MortalityArch Intern Med. 2010;170(18):1622-1631. doi:10.1001/archinternmed.2010.348

41. Elias M y col. Left ventricular mass, blood pressure, and lowered cognitive performance in the Framingham offspring. Hypertension 2007; 49:439,

42. Kaplan N y col. Clinical implications and treatment of left ventricular hypertrophy in hypertension. En: Bakris G editor. Uptodate 17.3. 2009. Disponible en URL: www.uptodate.com

43. Verdecchia P y col. Left ventricular mass and cardiovascular morbidity in essential hypertension: the MAVI study. J Am Coll Cardiol 2001; $38: 1829.2001 ; 104: 2039$.

45. Koren M y col. Role of left ventricular mass and geometry to morbidity and mortality in uncomplicated essentsial hypertension. Ann Intern Med 1991; 114:345.

46. Ruilope L y col. Left ventricular hypertrophy and clinical outcomes in hypertensive patients. Am J Hypertens 2008; 21:500.

47. Franz I y col. Time course of complete normalization of left ventricular hypertrophy during long-term antihypertensive therapy with angiotensin converting enzyme inhibitors. Am J Hypertens $1998 ; 11: 631$.

48. Okin P y col. Regression of electrocardiographic left ventricular hypertrophy during antihypertensive treatment and the prediction of major cardiovascular events. JAMA 2004; 292:2343.

49. Pierdomenico $S$ y col. Regression of echocardiographic left ventricular hypertrophy after 2 years of therapy reduces cardiovascular ris
50. National Institute for Health and Clinical Excellence Hypertension: management of hypertension in adults in primary care. June 2006.

51. Chobanian A y col. Seventh report of the Joint National Committee on Prevention, Detection, Evaluation, and Treatment of High Blood Pressure. Hypertension. 2003 Dec;42(6):1206-52. Epub 2003 Dec 1.

52. Mancia G y col.Guidelines for the Management of Arterial Hypertension: The Task Force for the Management of Arterial Hypertension of the European Society of Hypertension (ESH) and of the European Society of Cardiology (ESC). J Hypertens. 2007 Jun; 25(6):1105-87.

53. Whitworth J y col. World Health Organiz

sion. J Hypertens. 2003 Nov;21(11):1983-92.

54. Svärdsudd K y col. Hypertensive eye ground changes. Prevalence, relation to blood pressure and prognostic importance. The study of men borm in 1913.Acta Med Scand. 1978;204(3):159-67.

55. Wong T y col. Retinalmicrovascular abnormalities and their relationship with hypertension,cardiovascular disease, and mortality. Surv Ophthalmol. 2001;46:59-80.
56. Klein B y col. Prevalence of glaucoma. The Beaver Dam Eye Study. Ophthalmology. 1992 Oct;99(10):1499-504.

57. Witt $\mathrm{N}$ y col. Abnormalities of retinal microvascular structure and risk of mortality from ischemic heart disease and stroke. Hypertension. 2006 May;47(5):975-81. Epub 2006 Apr 3.

58. Pechin A y Lovazzano S. Recomendaciones sobre el manejo de la Diabetes. Evid Act Pract Ambul. 13(2). 60-63. Abr-Jun. 2010. Disponible en URL: http://www.evidencia.org/hitalba-pagina-

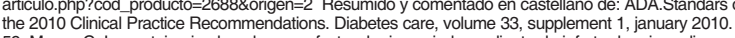

59. Musso C. La proteinuria elevada es un factor de riesgo independiente de infarto de miocardio, progresión de la nefropatía y mortalidad. Evid. Actual. Práct. Ambul; 13(3):92, Jul-Set 2010. Comentado de: Hemmelgam B y col. Relation between kidney function, proteinuria, and adverse outcomes. JAMA 2010;303(5):423-9. PMID: 20124537.

60 . Hemmelgayny col. Relation between kidney, proteinuria and adverse outcomes. JAMA 2010; 303 (5): $423-9$.

61. Diercks G y col. Microalbuminuria is independently associated with ischaemic electrocardiographic abnormalities in a large non-diabetic population. The PREVEND (Prevention of REnal and Vascular ENdstage Disease) study. Eur Heart J. 2000 Dec;21(23):1922-7.

62. Bakris. Microalbu Cartid intima cardiovascular disease. UpToDate 2010

64. Cobble M y col. Carotid Intima-Media Thickness: knowledge and application to everyday practice. Postgraduate Medicine. Volume 122. Issue 1. January 2010

64. Lombenz y col. Prediction of clinical cardiovascular events with carotid intima-media thickness. Circulation 2007;115:459-467.
65.

66. Bonow R. Should Coronary Calcium Screening Be Used in Cardiovascular Prevention Strategies? N Engl J Med 2009;361:990-7.

67. Newman A y col. Ankle-arm index as a predictor of cardiovascular disease and mortality in the Cardiovascular Health Study. The Cardiovascular Health Study Group. Arterioscler Thromb Vasc Biol 1999; 19:538-4.

68. Bluro I. El índice tobillo-brazo puede mejorar la predicción de eventos cardiovasculares. Evid. Actual. Práct. Ambul; 12(2): 56-57, Abr-Jun 2009. Comentado de: Ankle Brachial Index Collaboration. Ankle Brachial Index Combined With Framingham Risk Score to Predict Cardiovascular Events and Mortality. JAMA 2008; 300: 197-208. Disponible en URL: http://www.evidencia.org/hitalba-pagina-articulo.php?cod_producto=2525\&origen=2 (últi-
mo acceso 21/11/10).

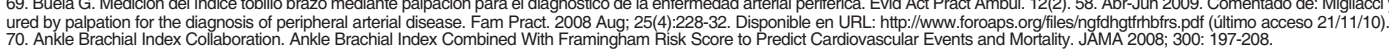

71. Schargrodsky H. CARMELA: Assessment of Cardiovascular Risk in Seven Latin American Cities. American Journal of Medicine (2008) 121, 58-65. 